\title{
El sueldo no es suficiente para atraer y retener a los mejores
}

\author{
Rosa María Fuchs Ángeles \\ International MBA por el Instituto de Empresa Business School, Madrid-España. Profesora e \\ investigadora del Departamento Académico de Administración de la Universidad del Pacífico, \\ Lima, Perú.

\section{Susana Sugano Sato} \\ MBA por la Escuela de Postgrado de la Universidad del Pacífico. \\ Profesora e investigadora del Departamento Académico de Administración de la Universidad del \\ Pacífico, Lima, Perú.
}

\begin{abstract}
Resumen
Las organizaciones afrontan el reto de atraer y retener al personal talentoso. En diversas instancias se hace referencia a la existencia de una guerra por el talento. Es así que la retención de los trabajadores ha dejado de trabajarse, exclusivamente, considerando solo la oferta de un buen sueldo. En este artículo se explora, en un inicio, el marco teórico que describe las principales herramientas de retención del talento que desarrollan las empresas, y que son consideradas como parte de la retribución total que reciben los trabajadores: compensaciones económicas, reconocimiento, planes de carrera y desarrollo del personal, retroalimentación, equilibrio vida profesional-vida personal, lazos sociales y generación de equipos, adecuada relación con el jefe y clima organizacional.
\end{abstract}

Posteriormente, se presentan los resultados que se obtuvieron mediante entrevistas con diez de las mejores empresas para trabajar del Perú, según el ranking del Great Place to Work Institute de 2007, sobre las herramientas de retención del talento que ponen en práctica. Finalmente, se presentan las conclusiones del estudio de casos.

\section{Palabras clave}

Compensación, atraer al talento, herramientas de retención, tendencias en compensaciones

\section{Introducción}

En el mundo de los negocios, actualmente, se reconoce la gran importancia del capital humano para alcanzar los objetivos organizacionales. En este contexto, la lucha por atraer y retener al personal talentoso lleva a las empresas a preocu- parse por diseñar sistemas atractivos de compensación. Los trabajadores parecen haber tomado conciencia de su papel estelar y no se conforman con un buen sueldo, sino que exigen cada vez mejores retribuciones. 
Las autoras discutirán y plantearán en el presente artículo los diferentes tipos de compensación que existen. Luego de haber consultado a las empresas reconocidas del Perú como los mejores lugares para trabajar, según el ranking elaborado por el Great Place to Work de 2007, presentarán los diferentes mecanismos que estas emplean para retribuir a sus empleados.

\section{Objetivos del estudio}

- Presentar los distintos mecanismos de retribución total que se emplean actualmente.

- Comprobar que las empresas peruanas más reconocidas por su gestión del talento humano se preocupan por emplear una mezcla atractiva de elementos de retribución para atraer y retener a sus trabajadores.

\section{Marco conceptual}

La retribución es una herramienta clave de la gestión de personas, pues influye en el comportamiento del trabajador, y es determinante para atraer, retener y motivar (Delgado et al. 2006). Según Gómez-Mejía et al. (2004), los componentes de la retribución total son: el sueldo base, los incentivos salariales y las prestaciones.

- Sueldo base: cantidad fija que recibe el trabajador de manera regular.

- Incentivos salariales: programas diseñados para recompensar el buen rendimiento de los empleados.

- Prestaciones: programas de seguros médicos, el facilitar el uso o adquisición de un automóvil, entre otros.

Las áreas de Gestión de Personas buscan que los sistemas de retribución total logren la equidad interna (que el trabajador considere que recibe una retribución justa dentro de la estructura de retribuciones de la propia empresa) y la equidad externa (que el trabajador considere que recibe una retribución justa con respecto al salario que pagan otra empresas por el mismo tipo de trabajo). Sin embargo, estas no son las dos únicas consideraciones. El sistema de compensación no puede crearse sin tomar en cuenta un factor fundamental: su alineamiento con los objetivos estratégicos organizacionales; es decir, que las empresas dejen de pagar por una posición específica o título de puesto y empiecen a recompensar a los empleados con bases en sus competencias individuales y en sus contribuciones laborales al éxito de la organización. La alineación puede tener un efecto positivo en la efectividad de la organización (Bohlander y Snell 2008).

Además de considerar los factores antes mencionados, las organizaciones viven en un contexto en el cual luchan por atraer y retener a personal talentoso (Fagalde 2008), por lo que al interior del sistema de retribución total debe encontrarse algo más que un buen sueldo. Las empresas, con el fin de evitar la salida del personal que ya ha formado y considera valioso, llevan a cabo diferentes mecanismos de retención, como ofrecer más dinero, realizar planes de carrera, ofrecer flexibilidad laboral, entre otros. Sin embargo, «la importancia, prioridad o valor que la gente talentosa le asigna a ciertos factores de su trabajo, no siempre son los mismos que los percibidos o detectados por la empresa» (Suárez 2007: 60). Esta es otra de las consideraciones para la organización. La gerencia de Recursos Humanos debe, entonces, entender y reconocer las diferentes necesidades del empleado para desarrollar planes adecuados de retención del talento.

Tres preguntas clave pueden ayudar al empleador a conocer las necesidades de los empleados más talentosos: ¿cuál es el reto más importante que tienes por delante?, ¿cuál es el obstáculo más grande que tienes para cumplir ese reto? y ¿qué puedo hacer yo (empleador) para ayudarte? Las respuestas darán una clara imagen de lo que busca, quiere, anhela, sueña y, en cierto modo, obliga al jefe a convertirse en un facilitador de sus objetivos (Sociedad Nacional de Minería, Petróleo y Energía 2008).

En el cuadro 1, sobre la base de la edad de los empleados, se describen sus distintas necesidades. 
Cuadro 1

Necesidades de los empleados según edades

\begin{tabular}{|c|c|}
\hline Etapa I & $\begin{array}{l}\text { - Los más jóvenes (digamos hasta los 23) dedican su atención a aprender y } \\
\text { desarrollarse. }\end{array}$ \\
\hline Etapa II & $\begin{array}{l}\text { El adulto en crecimiento (24-35) da prioridad a establecer una carrera y una } \\
\text { familia, y siente una presión significativa por mantener el equilibrio entre su } \\
\text { floreciente carrera y las necesidades de su familia. }\end{array}$ \\
\hline Etapa III & $\begin{array}{l}\text { - Hacia mitad de la vida (35-55) aumenta el deseo de liderar, de aconsejar, de } \\
\text { enseñar y de «tenerlo todo». }\end{array}$ \\
\hline Etapa IV & $\begin{array}{l}\text { - En la última etapa de la vida laboral (55-65) se reafirman los valores y el miedo a } \\
\text { un posible despido o a una adquisición de la compañía, presiona a muchos a } \\
\text { conservarlo que se ha conseguido, tal y como está. }\end{array}$ \\
\hline
\end{tabular}

Fuente: Adaptado de Marcos (2007)

Como se puede apreciar en el cuadro 1, resultaría importante agrupar a los distintos empleados de acuerdo con la etapa en la que se encuentren con el fin de establecer estructuras de retribución total flexibles.

Asimismo, las maneras de recompensar han evolucionado a lo largo de los años y se ha reforzado la idea de individualización de los planes de retribución. Chen y Hsieh (2006) describen la evolución del sistema de recompensas en el siglo XXI. Los cambios más importantes se describen en el cuadro 2.

Cuadro 2

Principales cambios en el sistema de recompensas

\begin{tabular}{|l|l|}
\hline \multicolumn{1}{|c|}{ Sistema tradicional de recompensas } & \multicolumn{1}{c|}{ Tendencias en las recompensas } \\
\hline $\begin{array}{l}\text { Sistema basado en los años de servicio del } \\
\text { empleado. }\end{array}$ & $\begin{array}{l}\text { Sistema basado en la contribución que haya } \\
\text { hecho el empleado en la creación de valor para la } \\
\text { empresa. }\end{array}$ \\
\hline $\begin{array}{l}\text { Pago solo en dinero (mecanismo extrínseco), no } \\
\text { duradero en el tiempo. }\end{array}$ & $\begin{array}{l}\text { Sistema de recompensa total, combinando } \\
\text { aspectos extrínsecos e intrínsecos valorados por } \\
\text { el empleado. }\end{array}$ \\
\hline Sistemas que tienen lugar una o dos veces al año. & $\begin{array}{l}\text { Los mecanismos de recompensas suceden de } \\
\text { manera constante y oportuna. }\end{array}$ \\
\hline $\begin{array}{l}\text { Solo existe un tipo de programa de recompensas } \\
\text { que se aplica a todos los trabajadores por igual. }\end{array}$ & $\begin{array}{l}\text { Se consideran las necesidades individuales de los } \\
\text { empleados. }\end{array}$ \\
\hline
\end{tabular}

Fuente: Chen y Hsieh (2006)

El cuadro anterior muestra cómo algunos factores -solo el dinero o los años de servicio- han dejado de tener relevancia frente a las necesidades y contribuciones individuales de los empleados.
Algunas de las preguntas que podrían ayudar al diseño de un plan adecuado de retribución se presentan en el cuadro 3. 
Cuadro No. 3

Cuestionamientos antes de diseñar un Plan de Recompensas

- ¿Es rentable como inversión? ¿Tiene un impacto medible y positivo en la productividad, la retención, la reputación corporativa, la calidad, la creatividad y/o el servicio al cliente?

- ¿Apoya a la cultura empresarial? ¿Respeta los valores de la empresa?

- ¿Hay otros beneficios que resulten atractivos para los empleados (sobre todo los mejores)? ¿Qué ofrecen otras organizaciones competidoras por el talento que necesita la organización?

- ¿Qué percepción tienen de su valor los empleados y los directivos? ¿Son igualmente positivas las soluciones adoptadas para todos los empleados o se deberían considerar colectivos distintos con necesidades distintas?

- ¿Qué imagen proyectan sobre nuestra empresa hacia el exterior? ¿Aumentan nuestra capacidad para atraer a los mejores candidatos?

- ¿Son una necesidad para nuestro negocio o simplemente una buena idea?

Fuente: Marcos (2007)

Visto lo anterior, las empresas deben diseñar el plan de retribución total considerando la visión estratégica de la empresa, la estructura de sus trabajadores y sus necesidades, y las contribuciones individuales de los mismos hacia el logro de objetivos.

Por otro lado, es fundamental incidir en la importancia del sistema de comunicación en las políticas de recompensas, puesto que una razón para el fracaso de dichos incentivos podría estar en la simple omisión de la información y la comunicación con los trabajadores de la empresa. «Quizá, las personas se estén marchando a otras organizaciones no porque les estemos pagando poco o mal, sino por el desconocimiento de todo aquello que la empresa les proporciona» (Usandizaga 2007: 19). Sería pues absurdo desarrollar un sistema de recompensas a la medida, que permita retener a los empleados más talentosos dentro de la organización y, sin embargo, perder el talento por el simple hecho de no comunicarles la inversión que hace la empresa en ellos.

Herramientas de retención

A continuación se presenta una lista de diferentes tipos de herramientas de retención, relacionadas con los componentes del concepto de re- tribución total (factores extrínsecos e intrínsecos), relevantes para ayudar a las empresas a retener a los empleados más talentosos.

\section{- Compensaciones económicas}

Es el pago que una persona recibe en la forma de sueldos, salarios, comisiones y bonos, entre otros. Si bien no es un factor determinante para la retención de los empleados, la empresa debe cuidar de mantener una equidad interna, así como una equidad externa (Mondy y Noe 2005).

\section{- Reconocimiento}

Es muy importante reconocer el buen desempeño de los trabajadores. En muchos casos, hasta un simple "gracias» basta para que el trabajador se sienta apreciado y valorado. EI hecho de no sentirse apreciado o reconocido es una de las principales causas por las cuales los empleados se van: « $35 \%$ de los trabajadores encuestados recientemente mencionan con frecuencia el reconocimiento de logros, como la recompensa no-monetaria más eficaz. Los individuos quieren saber que su trabajo es apreciado y hace una diferencia» (Teller Vision 2007: 5) (Traducción de las autoras). Es importante reconocer tanto el esfuerzo como el desempeño de los trabaja- 
dores. A las personas les gusta ser reconocidas en frente de sus colegas, es decir, que el reconocimiento sea público (Braham 1989) (Traducción de las autoras).

Si bien el reconocimiento es muy importante como herramienta de retención, este puede acarrear algunos problemas, si no se lleva a cabo de manera adecuada. Los programas de reconocimiento pueden perder su efectividad por una mala gestión. Si se les da «reconocimiento» a todos por igual, este pierde su significado. Si se le da a la persona equivocada, también pierde su poder. Por esta razón, se debe hacer un esfuerzo en identificar aquellos empleados que brindan mayor valor a la compañía, aquellos empleados talentosos, ya que son precisamente a ellos a quienes se les tiene que reconocer (Braham 1989). La empresa debe ser consistente en otorgar los reconocimientos y debe comunicar claramente las razones de los mismos.

- Planes de carrera y desarrollo del personal Para muchos empleados es importante saber que la empresa los va a desarrollar profesionalmente durante el tiempo que estén en la empresa, lo que irá acompañado de continuos ascensos, que les permitan desarrollar al máximo su potencial. «El desarrollo y oportunidades de carrera son un tema cada vez más valorado por las personas que acceden al mundo laboral. Iniciativas como la rotación en los puestos; las asignaciones internacionales o un plan de sucesiones estructurado y real, ayudan enormemente a la hora de atraer, retener y motivar el talento» (Usandizaga 2007: 19).

Muchos empleados ingresan a una compañía con el deseo de aprender y crecer en ella. La mayoría tiene expectativas de desempeñar funciones cada vez más desafiantes, por lo que exigen de la compañía la ayuda necesaria (mediante planes de entrenamiento) para poder desarrollarse como profesionales dentro de la misma.
La mayoría de las empresas tiene ya establecidos planes de sucesión, en los que se procura tener un backup ante el posible abandono de un empleado. Es decir, ya saben quién ascendería a un determinado puesto, en el caso de que ocurra la desvinculación de algún empleado. Es necesario conversar con los empleados para saber hasta dónde quieren llegar en la empresa y, sobre la base de estas expectativas, elaborar los respectivos planes de carrera y sucesión, de manera que pueda considerarse como una herramienta de retención efectiva.

Sin embargo, según una entrevista realizada a Cristina Simon, profesora del Instituto de Empresa de Madrid, el concepto de lealtad ha ido perdiendo fuerza con la llegada de cada generación, por lo que las empresas han cambiado la oferta de estabilidad por la de empleabilidad (Wharton.Universia.Net 2007). Esta última puede ser definida como la capacidad para encontrar, crear, conservar y enriquecer un trabajo, y pasar de uno a otro obteniendo a cambio una satisfacción personal, económica, social y profesional (Universia Perú 2007). Por lo tanto, los planes de desarrollo no solo están necesariamente ligados a las funciones que cumple el empleado, sino que, además, les permite generar habilidades para desempeñarse eventualmente fuera de la empresa.

- Retroalimentación

Todos los empleados tienen la necesidad de recibir información con relación a si el trabajo que realizan está bien hecho o existen algunos puntos que deban revisarse. De esta manera, el empleado puede sentirse encaminado hacia el logro de los objetivos, contando con el respaldo de su empleador. Si el empleado no recibe una retroalimentación, avanza a ciegas; si hubiese hecho algo mal, seguirá haciéndolo mal y si hace algo bien, no será reconocido de la manera adecuada.

La retroalimentación «no solo debe reservarse para casos de bajo desempeño y errores, sino también para reconocer fortalezas y lo- 
gros. Para que la retroalimentación sea efectiva, ésta debe ser oportuna, específica, propia del evaluador, entendible por el evaluado, provista de un clima de soporte, y necesariamente seguida de un plan de acción» (Gonzales 2006: 33).

- Equilibrio vida profesional/vidapersonal «El equilibrio vida personal/vida profesional o conciliación, se fundamenta en la flexibilidad. En una época en la que todas las organizaciones dicen estar enfocadas a resultados, no debería extrañarnos que se establezcan políticas destinadas a mejorar dicha flexibilidad » (Usandizaga 2007: 18-19).

En un ambiente laboral cada vez más involucrado con el estrés, los empleados valoran más el hecho de poder mantener una vida equilibrada en lo que a trabajo y vida personal se refiere. Las personas no solo se desempeñan como profesionales, sino también cumplen funciones dentro del núcleo familiar $y$ social, lo cual implica una serie de metas y sueños que desean lograr. Por ello, muchos trabajadores piden jornadas laborales más flexibles, lo cual por ninguna razón implica que los objetivos trazados no se vayan a cumplir. «Una vez comunicados los objetivos estratégicos, y la contribución que de cada uno de nuestros empleados se espera, el cómo se obtengan esos resultados en términos de hora de salida del lugar de trabajo, o del lugar donde se realiza (en la oficina, por medio del teletrabajo, entre otros), debería pasar no ya a un segundo plano, sino dejar de tenerse en cuenta, y empezar a valorar, tanto positiva como negativamente el logro de dichos objetivos y la aportación real del empleado a la compañía» (Usandizaga 2007: 19). Según Apgar (1999), treinta a cuarenta millones de estadounidenses trabajan lejos de su empresa o con base en su casa.

Al dar la suficiente libertad para que el empleado organice su tiempo de acuerdo con sus necesidades, se puede lograr incluso una mayor productividad. Según Capelli (2008), la clave para retener a la gente talentosa es encontrar el balance entre los intereses de los empleados y los empleadores. Es muy importante conocer los interés de los empleados y que estos sientan que la empresa trata de conocerlos (Lawler III 2008).

- Lazos sociales y generación de equipos Una herramienta de retención para las empresas es el fomento de relaciones sociales que unan a los miembros de la empresa. Esto se puede lograr mediante jornadas de integración y reuniones con motivos no-laborales, que permitan la interacción social entre los trabajadores. Una herramienta es el fomento del deporte en la organización. "Cada vez son más las empresas que sabiendo esto ponen en práctica esta tendencia e incluyen en los presupuestos internos las actividades que fomenten las relaciones intersociales» (Fernández 2006: 8). Asimismo, Capelli (2000) afirma que existen empleados que permanecen en la empresa motivados por las relaciones sociales creadas con sus compañeros de trabajo.

- Adecuada relación con el jefe

Una razón muy importante de la pérdida de talento en las empresas es la mala relación existente entre el jefe y el subordinado. El jefe, como líder de un equipo o un área específica, tiene ciertas responsabilidades y tareas que cumplir que fomentarán un adecuado clima laboral. En este ambiente, el empleado encontrará no solo bienestar, sino también todas las herramientas necesarias para llevar a cabo sus labores de una manera exitosa. « [...] que los ejecutivos, empleados y operarios estén contentos con la empresa con la que trabajan, no es responsabilidad sólo del gerente de recursos humanos sino de cada jefe, de cada persona que tiene supervisión directa sobre un grupo de profesionales» (Sociedad Nacional de Minería, Petróleo y Energía 2008: 21). De esta manera, se puede decir que el papel del líder es fundamental en el proceso de retención del talento. 
Además, «todas las encuestas relacionadas con la identificación de las principales razones por las que el mejor talento se va de una empresa tienen un elemento en común: la pérdida de credibilidad y confianza en sus líderes es la más importante de todas las razones» (Suárez 2007: 62).

\section{- Clima organizacional}

Adicionalmente a las herramientas mencionadas, se puede incluir al clima organizacional, que es el conjunto de variables que incide en la percepción (por lo tanto, es subjetivo) que tienen las personas del lugar donde desarrollan su actividad laboral. Existen diversos factores que influyen en él, entre los que se pueden mencionar: la formación, las expectativas de promoción, el sistema remunerativo y el grupo de trabajo (Córdova 2004). Esto supone que la combinación adecuada de las herramientas citadas haría que el clima organizacional sea más favorable y, por lo tanto, contribuya a que los trabajadores se sientan menos atraídos por dejar la empresa.

\section{Metodología}

Las autoras decidieron acercarse a diez de las mejores empresas para trabajar en el Perú, según el ranking del Great Place to Work Institute del 2007. Se realizaron entrevistas con los geren- tes de Recursos Humanos de cada una de ellas y se aplicó un cuestionario con el fin de conocer los diferentes mecanismos de retribución que utilizan para retener a su personal talentoso. En el cuadro 4 se muestran los sectores a los cuales pertenecen las empresas.

\section{Casos peruanos}

En las empresas peruanas también se ofrecen factores adicionales al sueldo como compensación al trabajador. A continuación, se podrá apreciar la opinión de las empresas entrevistadas respecto de las herramientas de retención mencionadas anteriormente. En los cuadros respectivos a cada factor de retención, se presenta la información obtenida de cada una de ellas.

\section{Compensaciones económicas}

Las compensaciones económicas desempeñan un papel relevante al momento de atraer nuevo personal. En promedio, las empresas encuestadas han mencionado que manejan sueldos competitivos de mercado, siendo superiores en el caso de las personas con mayor desempeño. Además, las remuneraciones se complementan con beneficios adicionales, como seguros, descuentos en acciones, bonos por desempeño, medios de comunicación y transporte, facilidad de préstamos, vacaciones adicionales, entre otros (véase el cuadro 5).

\section{Cuadro 4}

Sectores a los que pertenecen las empresas entrevistadas

\begin{tabular}{|l|c|}
\hline \multicolumn{1}{|c|}{ Sectores } & $\mathbf{N}^{\circ}$ de empresas \\
\hline Farmacéutico & 2 \\
Comercial & 1 \\
Financiero & 1 \\
Consumo masivo & 1 \\
Tecnología & 3 \\
Servicios & 2 \\
\end{tabular}

Elaboración propia. 
Cuadro 5

Compensaciones económicas en las empresas encuestadas

\begin{tabular}{|c|c|}
\hline Sector & Aplicación de la herramienta \\
\hline \multirow[t]{2}{*}{ Farmacéutico } & $\begin{array}{l}\text { El sueldo tiene mayor capacidad para atraer que para retener. Las compensa- } \\
\text { ciones son competitivas. Además, se otorgan beneficios como compra de } \\
\text { acciones con descuentos y programa de bonos de acuerdo con el desempeño. }\end{array}$ \\
\hline & $\begin{array}{l}\text { El sueldo es por lo menos igual al promedio del mercado o superior a este. } \\
\text { Además, se cuentan con beneficios como EPS cubierto al } 90 \% \text {, celular, } \\
\text { cafetería cubierta al } 70 \% \text {, uniformes y auto plan. }\end{array}$ \\
\hline Comercial & $\begin{array}{l}\text { El sueldo tiene mayor capacidad para atraer que para retener. Beneficios } \\
\text { adicionales: seguro de salud cubierto por la empresa, almuerzo y estaciona- } \\
\text { miento gratuitos en las instalaciones, gasolina para vendedores, automóviles } \\
\text { para los gerentes. }\end{array}$ \\
\hline Financiero & $\begin{array}{l}\text { Estructura salarial competitiva (3er cuartil del mercado financiero). Bolsas } \\
\text { de incremento salarial, utilizadas en casos de retención. Conscientes de las } \\
\text { distintas necesidades de las personas, por lo que cada trabajador puede } \\
\text { escoger dentro del sistema de beneficios las variables disponibles. }\end{array}$ \\
\hline Consumo masivo & $\begin{array}{l}\text { El sueldo tiene mayor capacidad para atraer que para retener. La empresa } \\
\text { tiene una política de aumentos anuales por mérito. En su mayoría, los } \\
\text { beneficios están relacionados con el desempeño (bonos y sueldos variables). }\end{array}$ \\
\hline \multirow[t]{3}{*}{ Tecnología } & $\begin{array}{l}\text { Otorga incentivos económicos competitivos. El trabajador tiene la posibilidad } \\
\text { de comprar acciones de la compañía. Otros beneficios: seguro médico con } \\
\text { póliza preferencial, estacionamiento gratis, celular y laptop, automóviles } \\
\text { para los gerentes. }\end{array}$ \\
\hline & $\begin{array}{l}\text { El dinero es considerado como un elemento que no motiva, pero sí } \\
\text { desmotiva. Se trabaja el concepto de diferenciación en la organización: se } \\
\text { le da más a quien más contribuye. Se revisan las posiciones salariales en } \\
\text { función del mercado, el desempeño y las habilidades de la persona. }\end{array}$ \\
\hline & $\begin{array}{l}\text { Para la empresa un buen sueldo es un elemento que busca la mayoria de } \\
\text { trabajadores peruanos. La empresa otorga mejores sueldos a quienes son } \\
\text { considerados empleados talentosos. } \\
\text { La empresa trasmite el significado de compensación no solo como sueldo, } \\
\text { sino como un paquete completo que incluye el sueldo fijo, los ingresos } \\
\text { variables, los beneficios, entre otros. Se apuesta por el incremento del } \\
\text { sueldo variable. Otros beneficios: estacionamiento gratis, seguro médico con } \\
\text { cobertura de } 3 \text { millones de dólares, seguro oncológico, programa de } \\
\text { automóvil para los vendedores mediante préstamos, automóviles para los } \\
\text { gerentes financiado al } 100 \% \text { por la empresa. }\end{array}$ \\
\hline \multirow[t]{2}{*}{ Servicios } & $\begin{array}{l}\text { La empresa considera que el sueldo sirve más para atraer que para retener al } \\
\text { empleado. La empresa otorga } 15 \text { días } \\
\text { adicionales a las vacaciones de ley, bonos de alimentación, préstamos de } \\
\text { libre disponibilidad, entre otros. }\end{array}$ \\
\hline & $\begin{array}{l}\text { La empresa mantiene la equidad externa, de acuerdo con el promedio del } \\
\text { mercado. Algunos de los beneficios que ofrece son: programas de becas de } \\
\text { estudio, pases para obras de teatro y conciertos, beneficios con las otras } \\
\text { empresas del grupo. }\end{array}$ \\
\hline
\end{tabular}

Elaboración propia sobre la base de las entrevistas realizadas. 


\section{Reconocimiento}

Acerca de los programas de reconocimiento, todas las empresas mencionan la importancia de tenerlos como parte de su estrategia. Los reconocimientos a sus mejores empleados tienen características económicas (bonos por actividades o acciones concretas, vales de consumo, becas de estudio) y no económicas (trofeos, menciones públicas, cartas a familiares), tal como se muestra en el cuadro 6.

\section{Cuadro 6}

Reconocimiento en las empresas encuestadas

\begin{tabular}{|c|c|}
\hline Sector & Aplicación de la herramienta \\
\hline \multirow[t]{2}{*}{ Farmacéutico } & $\begin{array}{l}\text { Programas de reconocimientos económicos y no económicos por } \\
\text { acciones puntuales, distintas al desempeño; por ejemplo, reducción de } \\
\text { la burocracia, vales de compra, placas de reconocimiento, fines de } \\
\text { semana en un lugar agradable. Programas en los cuales los empleados } \\
\text { pueden reconocer a sus compañeros, lo cual es compartido por medio de } \\
\text { la Intranet. }\end{array}$ \\
\hline & $\begin{array}{l}\text { Programas de reconocimiento para los trabajadores que «vivan» los } \\
\text { valores corporativos. Asimismo, se reconocen acciones destacadas del } \\
\text { mes, que han sido concretadas individual o grupalmente. }\end{array}$ \\
\hline Comercial & $\begin{array}{l}\text { Programas de reconocimiento al mejor vendedor, al mejor empleado del } \\
\text { mes, al mejor empleado del año. Reconocimientos públicos, premios } \\
\text { como vales para comer en un restaurante. }\end{array}$ \\
\hline Financiero & $\begin{array}{l}\text { Programas de reconocimiento formal y sistemático en todo nivel. } \\
\text { Reconocimientos en el nivel individual y de equipo: desempeño } \\
\text { destacado, años de servicio, deportistas, instructores internos de } \\
\text { capacitación. }\end{array}$ \\
\hline Consumo masivo & $\begin{array}{l}\text { Se premia a los colaboradores por acciones excepcionales, incluye una } \\
\text { carta que se envía a los familiares contándoles dicha experiencia. } \\
\text { Además, se realiza premiaciones a los empleados de acuerdo con el } \\
\text { tema trabajado en el año. }\end{array}$ \\
\hline \multirow[t]{3}{*}{ Tecnología } & $\begin{array}{l}\text { Premiación anual corporativa en reunión mundial, se premia al círculo } \\
\text { de excelencia. Asimismo, premiaciones trimestrales en el nivel de } \\
\text { Latinoamérica, con un incentivo económico. Reconocimientos por } \\
\text { acciones destacadas que no involucran premios económicos, sino } \\
\text { reconocimientos públicos regionales. }\end{array}$ \\
\hline & Se cuenta con programas de reconocimiento monetario y no monetario. \\
\hline & $\begin{array}{l}\text { Existe un programa de reconocimiento corporativo, por medio del cual } \\
\text { cuando un empleado realiza una acción sobresaliente, su gerente } \\
\text { puede entrar al sistema y, luego de describir el logro del empleado, } \\
\text { asignarle puntos, que luego se convierten en soles. Los puntos pueden } \\
\text { ir entre } 100 \text { y } 3.000 .\end{array}$ \\
\hline \multirow[t]{2}{*}{ Servicios } & $\begin{array}{l}\text { El reconocimiento oportuno puede darse ya sea mediante una llamada } \\
\text { telefónica o en reuniones especiales; entregando diplomas, becas de } \\
\text { estudio, bonos económicos; entre otros. }\end{array}$ \\
\hline & $\begin{array}{l}\text { La empresa desarrolla diversas iniciativas: reconocimiento al mejor } \\
\text { desempeño semestralmente, el mejor colaborador del mes, reconocimien- } \\
\text { to del cliente fantasma (semestralmente), reconocimiento a la mejor } \\
\text { jefatura y a la mejor gerencia (anualmente), reconocimiento a las } \\
\text { mejores áreas. No todos los premios son económicos, algunos consisten } \\
\text { en otorgar una medalla, un diploma y reconocerlos públicamente. }\end{array}$ \\
\hline
\end{tabular}




\section{Planes de carrera y desarrollo del personal}

El cuadro 7 muestra que los planes de carrera y desarrollo del personal es quizá la herramienta más trabajada por las empresas encuestadas. Una de ellas los considera como su principal herramienta de retención. Las empresas se preocupan de que los planes de carrera vayan atados a criterios como la evaluación del desempeño y del potencial; de esta forma, aseguran la línea de sucesión.

Cuadro 7

Planes de carrera en las empresas encuestadas

\begin{tabular}{|c|c|}
\hline Sector & Aplicación de la herramienta \\
\hline \multirow[t]{2}{*}{ Farmacéutico } & $\begin{array}{l}\text { Se prefiere contratar a personas que puedan desarrollarse en la empresa, antes que personas con experiencia } \\
\text { (dependiendo del puesto). Los empleados considerados talentosos son aquellos que tienen mayor posibilidad de } \\
\text { hacer línea de carrera. Para ello, se estructura un programa interno de rotación de puestos y sucesión. También } \\
\text { se incentiva la participación en proyectos o talleres, donde en principio solo interviene el comité gerencial. } \\
\text { Otras consideraciones son, por ejemplo, el pago de estudios de posgrado o la asistencia a capacitaciones } \\
\text { internacionales. La evaluación de desempeño contiene una parte cuantitativa y otra, cualitativa. }\end{array}$ \\
\hline & $\begin{array}{l}\text { Se prefiere desarrollar a las personas internamente. Se preocupa por que cada trabajador tenga un } \\
\text { programa de desarrollo personalizado. Herramienta efectiva para asegurar el plan de sucesión. Se evalúa } \\
\text { tanto el desempeño como el potencial. }\end{array}$ \\
\hline Comercial & $\begin{array}{l}\text { Líneas de carrera (horizontal y vertical) ya trazadas. Los empleados saben que dentro de un período } \\
\text { determinado, si cumplen con los requisitos, tendrán la posibilidad de ascender. }\end{array}$ \\
\hline Financiero & $\begin{array}{l}\text { Señalado como principal herramienta de retención. } \\
\text { Se invierte mucho en capacitación, existen planes de sucesión formalmente diseñados, posibilidad de } \\
\text { rápido crecimiento dentro de la organización. Se buscan personas con capacidad de aprendizaje (lo cual } \\
\text { indica su potencial). }\end{array}$ \\
\hline $\begin{array}{l}\text { Consumo } \\
\text { masivo }\end{array}$ & $\begin{array}{l}\text { Se prefiere contratar personas sin mayor experiencia con el fin de desarrollarlas internamente. Se cuenta } \\
\text { con un programa de trainees que realizan prácticas preprofesionales en las distintas áreas de la } \\
\text { organización. Se tiene definidas las líneas de carrera. Plan de desarrollo de líderes y programas de } \\
\text { coaching para aquellos que ocuparán cargos gerenciales, en el nivel local e internacional. Se considera } \\
\text { tanto el desempeño como el potencial de las personas. }\end{array}$ \\
\hline \multirow[t]{4}{*}{ Tecnología } & $\begin{array}{l}\text { Se mide el desempeño y el potencial de los trabajadores; y sobre esta base se define si la persona hará } \\
\text { carrera en la empresa. Se realiza una reunión especial a mediados de año para discutir los planes de } \\
\text { carrera de cada trabajador. Es importante que el trabajador este interesado en sus planes de desarrollo. } \\
\text { La propuesta de valor para el empleado consiste en brindar desarrollo profesional. }\end{array}$ \\
\hline & $\begin{array}{l}\text { Uno de los elementos más importantes en la propuesta de valor al empleado es el desarrollo de carrera. } \\
\text { Busca el desarrollo de la persona de manera holística. Esperan desarrollar competencias fundamenta- } \\
\text { les y competencias relacionadas con la posición específica. La Intranet es utilizada para estos fines } \\
\text { de desarrollo. Una vez al año, el empleado debe documentar su Plan Individual de Desarrollo (IDP- } \\
\text { Individual Development Plan), indicando cuáles son sus metas en el corto y largo plazo. } \\
\text { Se desarrollan planes de sucesión. Las oportunidades están abiertas a todos los trabajadores en el } \\
\text { ámbito mundial. } \\
\text { La empresa mide el desempeño y potencial de los trabajadores para identificar su personal más talentoso. }\end{array}$ \\
\hline & $\begin{array}{l}\text { Se evalúa el desempeño y potencial de los empleados. Asociado a este tema se manejan los planes de sucesión. } \\
\text { La propuesta de valor para los empleados de esta empresa es brindar un lugar donde los trabajadores } \\
\text { puedan desarrollar su carrera. }\end{array}$ \\
\hline & $\begin{array}{l}\text { Existe un programa de desarrollo de carrera abierto, cuya información se encuentra en la página web. El } \\
\text { primer paso consiste en una reunión del empleado con su jefe. Luego se definen los objetivos de } \\
\text { desarrollo profesional, que se establecen en el Performance Plan. }\end{array}$ \\
\hline \multirow[t]{2}{*}{ Servicios } & $\begin{array}{l}\text { Se evalúa el desempeño y potencial de los trabajadores. Existen posibilidades de hacer línea de carrera } \\
\text { en la empresa. También se manejan planes de sucesión. }\end{array}$ \\
\hline & $\begin{array}{l}\text { La propuesta de valor para los empleados de esta empresa consiste en el ofrecimiento de oportunidades } \\
\text { de desarrollo al interior de la misma. La empresa ofrece posibilidades de desarrollo de carrera. Los } \\
\text { colaboradores interesados, de manera voluntaria, se presentan para ser evaluados y comenzar a desarrollar } \\
\text { su línea de carrera. También pueden desarrollar su carrera en otras empresas del grupo. Para la empresa } \\
\text { esta es una herramienta de retención del talento. }\end{array}$ \\
\hline
\end{tabular}

Elaboración propia sobre la base de las entrevistas realizadas. 


\section{Retroalimentación}

Si bien algunas empresas no han indicado explícitamente las prácticas relacionadas con la retroalimentación (véase el cuadro 8), podría entenderse que existen reuniones de los em- pleados con los jefes para un adecuado alineamiento cuando se realiza la evaluación de desempeño y se determinan los planes de carrera y desarrollo.

Cuadro 8

Sistema de retroalimentación en las empresas encuestadas

\begin{tabular}{|l|l|}
\hline \multicolumn{1}{|c|}{ Sector } & \multicolumn{1}{c|}{ Aplicación de la herramienta } \\
\hline Farmacéutico & $\begin{array}{l}\text { Tres veces al año cada empleado se reúne con su jefe directo para una } \\
\text { sesión de retroalimentación acerca de su evolución en la empresa. }\end{array}$ \\
\hline Comercial & \begin{tabular}{l} 
Constantemente se llevan a cabo reuniones con los empleados. \\
\hline Financiero
\end{tabular} \\
$\begin{array}{l}\text { Desayunos de calidad, en donde los empleados realizan sugerencias. Las } \\
\text { mejores son premiadas. }\end{array}$ \\
\hline
\end{tabular}

Elaboración propia sobre la base de las entrevistas realizadas.

\section{Equilibrio vida profesional/vidapersonal}

Las empresas entrevistadas consideran este factor como importante para atraer y retener a los trabajadores. Fundamentalmente, se está trabajando en brindar un horario de trabajo flexible. Algunas organizaciones han desarrollado mecanismos específicos, como cartillas o cuponeras

\section{Cuadro 9}

Equilibrio vida profesional/vida personal en las empresas encuestadas

\begin{tabular}{|c|c|}
\hline Sector & Aplicación de la herramienta \\
\hline Farmacéutico & $\begin{array}{l}\text { Horario flexible, se puede trabajar desde la casa (dependiendo del puesto), } \\
\text { flexibilidad para el otorgamiento de permisos. Existe una persona dedicada } \\
\text { exclusivamente a velar por el bienestar de los trabajadores, procurando que } \\
\text { mantengan un adecuado equilibrio entre la vida profesional y la personal. } \\
\text { Se organizan talleres ligados al desarrollo personal y ocio (marketing personal, } \\
\text { cata de vinos, entre otros), así como reuniones en donde los empleados pueden } \\
\text { invitar a su familia. }\end{array}$ \\
\hline Comercial & $\begin{array}{l}\text { Taller de manejo de estrés. Horarios flexibles, se trabaja por objetivos. Se } \\
\text { fomenta el trabajo desde casa, dependiendo del puesto de trabajo. }\end{array}$ \\
\hline Financiero & $\begin{array}{l}\text { Salida temprana los viernes, horario flexible, involucramiento de familiares en } \\
\text { actividades que organiza la empresa, cuponera de tiempo libre. }\end{array}$ \\
\hline Consumo masivo & $\begin{array}{l}\text { Horario flexible y trabajo desde la casa (dependiendo del puesto). Trabajo por } \\
\text { objetivos. }\end{array}$ \\
\hline Tecnología & $\begin{array}{l}\text { Se trabaja sobre la base de objetivos. Los miembros de la organización son libres } \\
\text { para manejar su tiempo, en coordinación con el gerente. La empresa considera } \\
\text { importante crear programas de flexibilidad. }\end{array}$ \\
\hline
\end{tabular}

de tiempo libre. La mayoría de las empresas entrevistadas propone el trabajo desde casa, siempre que sea posible y que la comunicación del trabajador con su jefe sea permanente. El detalle se puede apreciar en el cuadro 9 . 


\begin{tabular}{|l|l|}
\hline \multirow{7}{*}{ Servicios } & $\begin{array}{l}\text { Una de las principales estrategias de esta empresa es la flexibilidad. La empresa } \\
\text { considera que permitir que las personas organicen su tiempo y que puedan estar } \\
\text { en los momentos importantes de su vida personal y profesional es lo que otorga el } \\
\text { equilibrio. Los trabajadores deben coordinar con sus gerentes estas opciones. }\end{array}$ \\
\cline { 2 - 3 } & $\begin{array}{l}\text { Se cuenta con un programa de balance entre la vida personal y el trabajo. } \\
\text { Algunos trabajadores pueden trabajar desde su casa. La empresa es muy flexible } \\
\text { en cuanto a permisos. Estos esfuerzos se consideran como una importante } \\
\text { herramienta de retención. }\end{array}$ \\
\hline La empresa considera este aspecto como altamente valorado por los trabajadores. \\
En algunos casos, los trabajadores administrativos pueden trabajar desde su casa. \\
La empresa considera este aspecto como altamente valorado por los trabajadores. \\
En algunos casos, los trabajadores administrativos pueden trabajar desde su casa. \\
La empresa considera este factor como un mecanismo de retención. \\
Los trabajadores reciben una cartilla de tiempo libre, que les permite la \\
posibilidad de disponer de tiempo libre para los propósitos que consideren \\
conveniente.
\end{tabular}

Elaboración propia sobre la base de las entrevistas realizadas.

\section{Lazos sociales y generación de equipos}

Algunas de las empresas entrevistadas desarrollan campeonatos deportivos, celebran las fechas especiales del año-como día del padre y de la madre, Navidad- dedican un día para la familia, entre otros. Las autoras consideran que el hecho de que no todas las empresas hayan mencionado estas activi- dades puede deberse a que no las consideran relevantes como herramientas de retención del talento, sino como actividades de integración. El efecto de desarrollar estas actividades debe estar siendo medido en la evaluación de clima organizacional. El cuadro 10 muestra los resultados encontrados.

\section{Cuadro 10}

Lazos sociales y generación de equipos en las empresas encuestadas

\begin{tabular}{|c|l|}
\hline \multicolumn{1}{|c|}{ Sector } & \multicolumn{1}{c|}{ Aplicación de la herramienta } \\
\hline Comercial & $\begin{array}{l}\text { Anualmente hay una convención de ventas, asiste toda la empresa y el } \\
\text { viaje es cubierto por ella. } \\
\text { Celebraciones de bienvenida, cumpleaños, Halloween, Navidad. También } \\
\text { en ocasiones se incluye a la familia del trabajador. }\end{array}$ \\
\hline Consumo masivo & $\begin{array}{l}\text { El clima laboral positivo contribuye a que las personas se sientan como en } \\
\text { familia. Se realizan convenciones anuales en donde participan todas las } \\
\text { áreas de la empresa. }\end{array}$ \\
\hline Tecnología & $\begin{array}{l}\text { Existen actividades, como celebraciones de las fechas especiales del año, } \\
\text { que contribuyen a la creación de un espíritu de grupo. Celebran un día de } \\
\text { la familia. }\end{array}$ \\
\hline Servicios & $\begin{array}{l}\text { Se ha desarrollado un programa para que los empleados se conozcan } \\
\text { mejor. Los empleados se presentan ante sus compañeros, describen su } \\
\text { aporte a la empresa y expresan tres cosas que les gusten de la empresa y } \\
\text { tres que podrían mejorarse. }\end{array}$ \\
\cline { 2 - 3 } & $\begin{array}{l}\text { Existe un programa por el cuando un compañero atraviesa un problema, } \\
\text { los demás pueden participar en un concurso de baile interno para ayudarlo } \\
\text { a afrontarlo. También existen campeonatos deportivos. }\end{array}$ \\
\hline
\end{tabular}

Elaboración propia sobre la base de las entrevistas realizadas. 
Adecuada relación con el jefe

Solo algunas de las empresas entrevistadas opinaron sobre este tema. Probablemente, la importancia del jefe en relación con el comportamiento de sus empleados sea uno de los factores de man conciencia. Sin embargo, se puede apreciar

Cuadro 11

Adecuada relación con el jefe en las empresas encuestadas

\begin{tabular}{|l|l|}
\hline \multicolumn{1}{|c|}{ Sector } & \multicolumn{1}{c|}{ Aplicación de la herramienta } \\
\hline Farmacéutico & Política de puertas abiertas. \\
\hline Comercial & Política de puertas abiertas. \\
\hline Financiero & $\begin{array}{l}\text { Es responsabilidad de cada jefe velar por los miembros más talentosos de } \\
\text { su equipo. }\end{array}$ \\
\hline Consumo masivo & $\begin{array}{l}\text { "Plan de líderes», permite desarrollar las habilidades de liderazgo necesa- } \\
\text { rias para la gestión de los empleados. }\end{array}$ \\
\hline Tecnología & $\begin{array}{l}\text { Para que los jefes realicen una buena gestión de sus equipos, se les brinda } \\
\text { herramientas adecuadas; entre ellas, el coaching. } \\
\text { Se realiza una encuesta de retroinformación con los gerentes cada seis } \\
\text { meses. Los trabajadores opinan sobre ellos. }\end{array}$ \\
\hline \multirow{2}{*}{ Servicios } & $\begin{array}{l}\text { Se evalúa el trabajo del gerente mediante un programa en el cual los } \\
\text { trabajadores dan retroalimentación anónima a sus gerentes. } \\
\text { Cada seis meses, los trabajadores tienen la oportunidad de dar retroalimen- } \\
\text { tación a sus jefes. }\end{array}$ \\
\hline
\end{tabular}

Elaboración propia sobre la base de las entrevistas realizadas.

\section{Clima organizacional}

Las empresas entrevistadas son conscientes de la importancia que un buen clima organizacional tiene al momento de atraer y retener al personal talentoso. Además de considerar la medición del

clima periódicamente, desarrollan actividades para generar un lugar agradable para trabajar. Algunas de las prácticas son muy creativas, tal como se puede apreciar en el cuadro 12.

Cuadro 12

Clima organizacional en las empresas encuestadas

\begin{tabular}{|c|c|}
\hline Sector & Aplicación de la herramienta \\
\hline Farmacéutico & Se menciona como factor considerado al momento de seleccionar un lugar para trabajar. \\
\hline Financiero & Se menciona como factor considerado al momento de seleccionar un lugar para trabajar. \\
\hline Consumo masivo & Se menciona como factor considerado al momento de seleccionar un lugar para trabajar. \\
\hline \multirow[t]{2}{*}{ Tecnología } & $\begin{array}{l}\text { Se realiza una encuesta de clima organizacional anualmente. Se obtiene un índice de salud. } \\
\text { Algunas de las actividades que desarrollan: masajes y desayunos en épocas de cierre del año } \\
\text { fiscal (período con mucho estrés), un espacio para actividades recreativas, celebraciones de } \\
\text { Navidad, Halloween, Día de la Familia. } \\
\text { Se ha creado un servicio de transporte para los trabajadores que viven lejos de la empresa. }\end{array}$ \\
\hline & $\begin{array}{l}\text { Considera que es importante gestionarlo adecuadamente. Se evalúa el clima mediante la } \\
\text { encuesta Global Pulse Survey. }\end{array}$ \\
\hline
\end{tabular}




\begin{tabular}{|l|l|}
\hline \multirow{3}{*}{ Servicios } & Se realiza una encuesta de clima laboral anualmente. \\
\hline & $\begin{array}{l}\text { La empresa considera que este factor es decisivo cuando los trabajadores peruanos } \\
\text { deciden trabajar en una empresa. } \\
\text { Su propuesta de valor para los empleados contempla un excelente lugar para trabajar como } \\
\text { ofrecimiento. }\end{array}$ \\
\cline { 2 - 3 } & $\begin{array}{l}\text { La empresa considera que contar con un buen clima laboral es una herramienta de } \\
\text { retención del talento. } \\
\text { Entre las actividades que desarrolla se tienen: bailes coreográficos para ayudar a algún } \\
\text { compañero que necesite ayuda, campeonatos deportivos, celebraciones del día de la } \\
\text { amistad, día de la madre y del padre, fiesta de fin de año, Halloween y Navidad, un taller } \\
\text { para «recargar las pilas». } \\
\text { La empresa realiza trimestralmente evaluaciones de clima organizacional. }\end{array}$ \\
\hline
\end{tabular}

Elaboración propia sobre la base de las entrevistas realizadas.

\section{Resultados y conclusiones}

- Las empresas entrevistadas se preocupan por atraer y retener a su personal talentoso. En muchos casos, prefieren desarrollarlas desde los puestos iniciales en la organización.

- Las empresas peruanas listadas como las mejores para trabajar. en el año 2007, aplican una combinación de mecanismos de retribución para retener a su personal. No se basan exclusivamente en una remuneración competitiva.

- Si bien las remuneraciones son importantes para atraer a personas que desean trabajar en una determinada empresa, no son lo primordial al momento de retener a las personas más valiosas.

- Los mecanismos de reconocimiento y prácticas que permitan un adecuado balance entre la vida personal-vida profesional son utilizados por las empresas entrevistadas. Varias de las empresas están orientadas a los objetivos, por lo que el lugar donde cumplen sus funciones (espacio físico) pasa a un segundo plano (dependiendo del puesto de trabajo). 


\section{Referencias}

APGAR, M.

1999 «El centro de trabajo alternativo: cómo cambiar la forma y el lugar donde trabaja la gente». En: Harvard Business Review: Dirigir personas en la empresa. Bilbao, España: Ediciones Deusto, pp. 175-219.

BRAHAM, J.

1989 «A rewarding place to work». En: Industry Week, vol. 238, № 18, pp. 15-19.

BOHLANDER, G. y S. SNELL

2008 Administración de recursos humanos. 14a edición. México D.F.: Thompson Editores.

CAPELLI, P.

2008 «Talent Management for the Twenty-first Century». En: Harvard Business Review, vol. 86, No 3, pp. 74-81.

2000 «Market Driven Approach to Retaining Talent». En: Harvard Business Review, vol. 78, No 1, pp. 103-111.

CHEN, H. M. e Y. H. HSIEH

2006 «Key Trends of the Total Reward System in the 21st Century». En: Compensation and Benefits Review, vol. 38, № 6, pp. 64-70.

CÓRDOBA, A.

2004 «Clima laboral». En: VIZCAíNO, Eduardo (ed.). Expertos en personas. Madrid: Pearson Educación, S.A., pp. 143-158

DELGADO, M. I.; L. GÓMEZ, A. M. ROMERO y E. VÁSQUEZ

2006 Gestión de recursos humanos: del análisis teórico a la solución práctica. Madrid: Editorial Pearson Prentice Hall.

FAGALDE, A.

2008 «Enfrente la crisis de talentos y consiga los empleados que su empresa necesita». En: Conocimiento y Dirección, $\mathrm{N}^{\circ}$ 77, pp. 12

\section{FERNÁNDEZ, A.}

2006 «Deporte y trabajo: una sana y productiva combinación». En: E-Deusto, № 56, pp. 6-10.
GOMEZ-MEJÍA, L. R.; D. BALKIN y R. CARDY

2001 Dirección y gestión de recursos humanos. $3^{\text {a }}$ edición. Madrid: Editorial Prentice Hall.

\section{GONZALES, A.}

2006 «Feedback efectivo». En: Business Negocios en el Perú, vol. 12, №138, p. 33.

Great Place to Work ${ }^{\circledR}$ Institute

2007 «Best Companies in Peru» (Las 25 mejores empresas para trabajar en el Perú). <http:// www.greatplacetowork.com/best/list-pe2007.htm>.

LAWLER III, E.

2008 «Why are we Losing All our Good People». En: Harvard Business Review, vol. 86, $\mathrm{N}^{\circ} 6$, pp. 41-51.

MARCOS, S.

2007 « ¿Podemos seguir pensando que el dinero todo lo compra?». En: E-Deusto, № 65, pp. 14-20.

MONDY, R. W. y R. M. NOE

2005 Administración de recursos humanos. Novena edición. México: Pearson Educación.

\section{SOCIEDAD NACIONAL DE MINERÍA, PETRÓLEO Y} ENERGÍA

2008 "¿Cómo retener el talento en un mercado competitivo?». En: Desde Adentro, № 54 , pp. 18-21.

\section{SUÁREZ, L.}

2007 «La batalla por la retención del talento». En: Business Negocios en el Perú, vol. 13, № 14, pp. 60-62.

\section{TELLER VISION}

2007 «Recognition of Achievements Ranks as Top Employee Motivator», pp. 5-6. 
UNIVERSIA PERÚ

2007 «Competencias profesionales: claves de la empleabilidad». 2 de julio. <http:// www.universia.edu.pe/noticias/principales/ destacada . php?id=60366> .

USANDIZAGA, I.

2007 «El valor de la recompensa total en la guerra por el talento». En: E-Deusto, Nº61, pp. 14-19.

\section{WHARTON.UNIVERSIA.NET}

2007 «Duelo generacional en las empresas». En: Universia Knowledge@Wharton (revista digital). 18 de abril. <http:// www. wharton. universia.net/ index.cfm?fa=viewArticle\&id=1330>. 